\title{
Service Implementation by Phoniatricians during a Pandemic Emergency: A Questionnaire-based Survey
}

\author{
Original \\ Article \\ Reham Abdelwakil Ibrahim ${ }^{1}$,Tamer Abou-Elsaad ${ }^{2}$ \\ Phoniatric Unit, Department of Otorhinolaryngology, Faculty of Medicine, ${ }^{1}$ Assiut \\ University, Assuit, ${ }^{2}$ Mansoura University, Mansoura, Egypt.
}

\begin{abstract}
Objective: Providing a risk-assessment framework that guides Phoniatric clinical services delivery and also identify patients for whom these services should be prioritized during pandemics.

Design: Cross-sectional study.

Settings: A questionnaire-based survey was designed and directed to the phoniatricians based on their professional experience, work environment, and institutional resources. It was distributed online through various Phoniatric societies during the first wave of the COVID-19 pandemic. The questionnaire included grading of Phoniatric clinical services, methods of service delivery, triaging of patients/complaints according to morbidity, and demographic characteristics.

Main outcome measures: Recognition of the high acuity Phoniatric clinical services that should be delivered during pandemics as well as triaging the patient/complaint according to morbidity.

Results: The majority of Phoniatric clinical services are considered low acuity services except for the voice and swallowing disorders diagnostics. High acuity services include newborn hearing screening and rehabilitative services of swallowing disorders.

Conclusion: Most phoniatric services can be safely postponed during pandemics except for voice and swallowing disorders diagnostics and treatments. Consideration of tele-practice during pandemics.
\end{abstract}

Key Words: COVID-19, implemented services, phoniatricians, infective outbreak, pandemic survey.

Received: 22 June 2021, Accepted: 19 August 2021

Corresponding Author: Reham Abdelwakil Ibrahim, Department of Otorhinolaryngology, Faculty of Medicine, Assiut University, Assiut, Egypt, Tel.: 00201069984894, E-mail: : rehamabdelwakil@aun.edu.eg

ISSN: 2090-0740, 2021

\section{KEY POINTS OF THE STUDY}

1. Statements addressing Phoniatrics service delivery guidelines and recommendationsduring the COVID-19 pandemic are limited and based on experts' opinions.

2. This study aims to provide a risk-assessment framework that guides phoniatric clinical services delivery and also to identify critical patients during infection outbreaks using a questionnaire-based survey directed to the phoniatricians in multiple areas of the world.

3. Most Phoniatrics services can be safely postponed during pandemics except for voice and swallowing disorders diagnostics and treatments.

4. Dysphonic patients with airway compromiseand patients with swallowing and feeding difficulties are considered urgent patients.

5. Tele-practice should be considered as an alternative route for delivering the services.

\section{INTRODUCTION}

Phoniatrics is the medical field for communication and swallowing disorders, concerned with functions and diseases of voice, speech, language, hearing, and swallowing. The "Coronavirus disease 2019" was first reported in Wuhan, Hubei province, China, and has spread exponentially, resulting in a worldwide outbreak and a significant burden on the finite resources of many healthcare systems ${ }^{[1]}$. Reports from several scientific institutions stated that ENT specialists/Phoniatricians are at an increased risk of contracting the coronavirus/ SARS-CoV2 infection due to the presence of the virus extensively in the nasal and pharyngeal cavities of infected individuals ${ }^{[2,3]}$. The infection might be transmitted via procedures like nasoendoscopies or laryngoscopies that carry the risk of disseminating the virus through aerosol particles loaded with the virus hence, the nomenclature aerosol-generating procedures (AGPs) or by direct contact with contaminated surfaces during the patient 
confrontation $^{[3]}$. Accordingly, several phoniatric outpatient clinics deferred offering their patients the required clinical services to preserve their health resources or protect themselves from being infected. The only available formal guideline related to Phoniatric practice during the COVID-19 pandemic has been presented by the Union of European Phoniatricians (UEP) and included the opinions of a small number of experts Phoniatricians. Therefore, this questionnaire-based survey's main aimwas to gather Phoniatricians' perspectives on implementing Phoniatric services during the pandemic in a large cohort. This is to provide evidence-based guidelines to guide the provision of clinical Phoniatric services during the current outbreak or any future infectious outbreak.

\section{PATIENTS AND METHODS}

\subsection{Design:}

Cross-sectional study

\subsection{Settings:}

The authors reviewed the available guidelines and recommendations related to Phoniatric and ENT practices during the COVID-19 pandemic to guide the survey development. The authors designed a questionnairebased survey directed to the phoniatricians to inspect their clinical service applications during the pandemic. The questionnaire was distributed through the web on Google Forms to various Phoniatric societies and was available for answers from the $25^{\text {th }}$ of June till the $10^{\text {th }}$ of July, 2020. Participation in the questionnaire was entirely voluntarilyand self-administered. Both purposes and components of the questionnaire were illustrated before hand, and data of the respondents were kept anonymous.

The questionnaire was composed of four sections; Section I included grading of all domains implied in Phoniatric practice based on their acuity. Each domain consisted of three parts; non-interventional, interventional diagnostic, and treatment/rehabilitative services. Section IIincluded service delivery. Section III included classification of patients according to morbidity intoelective, semi-elective, semi-urgent, or urgent. Section IV included the demographic characteristics of the respondent.

All questions were in English and participants responded by choosing a single answer to each question. The questionnaire took an average of 10-15 minutes to complete. The authors presented the questionnaire to three experienced phoniatriciansother than the authors as a pilot study to evaluate the questionnaire in terms of the feasibility of answering; duration spent to be answered, fulfillment of the intended goals (face validity), coverage of all aspects of Phoniatric practice (content validity); and additional suggestions that might improve the questionnaire. The comments were considered, and the amendments were added to the questionnaire accordingly. Their responses were, however, not included in the statistical analysis.

Our research complied with human studies guidelines and was conducted ethically following the World Medical Association Declaration of Helsinki. The survey carried no risk to participants, and it was basically directed to scrutinize Phoniatric clinical practice in the current pandemic situations without affecting it in any way. Therefore, it was qualified for exemption from the Institution Review Board (IRB)'s approval (Blinded for review).

\section{Statistical analysis:}

Data from all responses were exported to SPSS version 22 (Statistical Package for Social Science) for Windows. Each grade/category was assigned a score, and the total scores were calculated. Descriptive statistics were presented as number, percentage, mean, and standard deviation. Independent samples t-test was used to compare between quantitative variables of parametric data. Pearson correlation test was done to measure the correlation between quantitative variables. P-value considered statistically significant when $P<0.05$.

\section{Main outcome measures:}

The recognition of the high acuity Phoniatricclinical services and the identification of the Phoniatric critical patients/complaints during pandemics.

\section{RESULTS}

All received responses are included in the statistical analysis, and none of them was incomplete. The majority of respondents had sufficient years of experience in practice, so we considered their responses reliable.

\subsection{Survey descriptive statistics:}

\section{Demographic data:}

One hundred five responses are received.The highest representation $(96,91.4 \%$ ) was from Egypt, while 8.6\% was from other countries. The respondents were mainly females $(90,85.7 \%)$, and $15(14.3 \%)$ were males. More than half $(56,53.3 \%)$ lied in the age group $30-40$ years and had $3-10$ years $(39,37.1 \%)$, or $10-20$ years $(37,35.2 \%)$ of professional experience. Over half worked in academic institutions $(58,55.2 \%)$, mostly included to treat COVID-19 cases $(67,63.8 \%)$. Nearly half $(48,45.7 \%)$ were provided with the necessary PPE by their institutions, and most of them $(96,91.4 \%)$ had access to technical support tools.

\section{Implemented services:}

The majority of phoniatricians graded the noninterventional diagnostic services of voice disorders grade one; the highest number observed in the voicerelated quality of life questionnaires $(76,72.4 \%)$ and the lowest in the diagnostic interview $(49,46.7 \%)$. However, the interventional diagnostic services of voice disorders were mostly considered grade two. This was also noted 
in phonosurgeries, except the office-based vocal fold injection were considered grade one. The rehabilitative services were also considered grade one.

Most respondents graded the non-interventional diagnostic services of speech, language and hearing disorders as grade one. Likewise, Language and speech therapies and alternative/augmentative communication. However, most contributors considered the interventional diagnostic services grade2. Similarly, the rehabilitation of the hearing disabled and prosthesis application were considered grade $2(50,47.6 \%)$ and $(56,53.3 \%)$ respectively.

The majority graded the non-interventional diagnostic services of swallowing disorders grade 2 , especially the nutritional evaluation $(49,46.7 \%)$; yet, the swallowingrelated quality of life questionnaires was considered grade one $(57,54.3 \%)$. The interventional diagnostic services of swallowing disorders were also rated grade 2 with the highest rates noted in the videofluoroscopic swallow study (VFSS); $(55,52.4 \%)$ and the lowest in the flexible endoscopic evaluation of swallowing (FEES); (49, 46.7\%). Swallowing therapy was equally assigned grades 2 and $3(45,42.9 \%)$; however, dietetic/nutritional therapy was mostly considered grade 3 (44, 41.9\%). The treatment services, e.g.,UES botulinum toxin injection, were considered grade1.Notably that the instrumental diagnostic services in all domains were mostly considered grade1 (Table1)

\section{Service delivery:}

The majority of respondents chose telepractice as a delivery approach for most Phoniatric services (Table 2).

Table 1: Descriptive statistics; implemented services/treatment

\begin{tabular}{|c|c|c|c|c|c|c|}
\hline \multirow{2}{*}{ Implemented services/treatment } & \multicolumn{2}{|c|}{ Grade $1^{1}$} & \multicolumn{2}{|c|}{ Grade $2^{2}$} & \multicolumn{2}{|c|}{ Grade $3^{3}$} \\
\hline & No. & $\%$ & No. & $\%$ & No. & $\%$ \\
\hline \multicolumn{7}{|l|}{ I. Voice Disorders } \\
\hline \multicolumn{7}{|l|}{ Non-interventional diagnostic services } \\
\hline Diagnostic interview & 49 & 46.7 & 38 & 36.2 & 18 & 17.1 \\
\hline Perceptual evaluation of voice & 52 & 49.5 & 38 & 36.2 & 15 & 14.3 \\
\hline Voice-related quality of life questionnaire & 76 & 72.4 & 21 & 20.0 & 8 & 7.6 \\
\hline Voice recording & 70 & 66.7 & 24 & 22.9 & 11 & 10.5 \\
\hline \multicolumn{7}{|l|}{ Interventional diagnostic services } \\
\hline Indirect laryngoscopy & 34 & 32.4 & 51 & 48.6 & 20 & 19.0 \\
\hline Rigid video/digital laryngostroboscopy & 25 & 23.8 & 56 & 53.3 & 24 & 22.9 \\
\hline Flexible transnasal video/digital laryngostroboscopy & 20 & 19.0 & 62 & 59.0 & 23 & 21.9 \\
\hline Instrumental diagnostic measures; EMG and EGG & 75 & 71.4 & 25 & 23.8 & 5 & 4.8 \\
\hline \multicolumn{7}{|l|}{ Rehabilitative Services } \\
\hline Voice therapy & 64 & 61.0 & 32 & 30.5 & 9 & 8.6 \\
\hline Electrolarynx rehabilitation & 59 & 56.2 & 37 & 35.2 & 9 & 8.6 \\
\hline \multicolumn{7}{|l|}{ Phonosurgery/ surgery } \\
\hline Suspension microlaryngoscopic vocal fold surgery; exophytic lesions & 30 & 28.6 & 49 & 46.7 & 26 & 24.8 \\
\hline Suspension microlaryngoscopic vocal fold surgery; intracordal lesions & 29 & 27.6 & 52 & 49.5 & 24 & 22.9 \\
\hline Laryngeal framework surgery; approximation, expansion, tensioning and relaxation & 40 & 38.1 & 43 & 41.0 & 22 & 21.0 \\
\hline Office-based transoral vocal fold injection & 51 & 48.6 & 42 & 40.0 & 12 & 11.4 \\
\hline Office-based transcutaneous vocal fold injection & 46 & 43.8 & 45 & 42.9 & 14 & 13.3 \\
\hline Application of voice prosthesis & 40 & 38.1 & 46 & 43.8 & 19 & 18.1 \\
\hline \multicolumn{7}{|l|}{ II. Speech, language and hearing disorders } \\
\hline \multicolumn{7}{|l|}{ Non-interventional diagnostic services } \\
\hline Diagnostic interview including family tree in familial hearing disorders & 65 & 61.9 & 31 & 29.5 & 9 & 8.6 \\
\hline Developmental questionnaires & 76 & 72.4 & 18 & 17.1 & 11 & 10.5 \\
\hline Evaluation of verbal and amp; non-verbal communication & 54 & 51.4 & 38 & 36.2 & 13 & 12.4 \\
\hline Evaluation of speech perception and production & 58 & 55.2 & 37 & 35.2 & 10 & 9.5 \\
\hline
\end{tabular}


Estimation of cognitive and developmental age

Evaluation of reading and writing

Interventional diagnostic services

Neurological examination

Speech and oral motor examination

Ear endoscopy and microscopy

Newborn hearing screening

Audiometric and electro-physiologic testing

Fiberoptic evaluation of velopharyngeal function

Instrumental diagnostic measures; acoustic and aerodynamic

\section{Rehabilitative services}

Language and speech therapy

Rehabilitation of hearing disabled

Augmentative and alternative communication

Treatment services

Prosthesis application; palatal lift or speech prosthesis

III. Swallowing Disorders

\section{Non-interventional diagnostic services}

Diagnostic interview

Bedside evaluation of swallowing

Nutritional evaluation

Swallowing-related quality of life questionnaires

\section{Interventional diagnostic services}

Flexible Endoscopic Evaluation of swallowing (FEES)

Videofluoroscopic Evaluation of Swallowing (VFES)

\section{Rehabilitative services}

Swallowing therapy

Dietetic and nutritional therapy

\section{Treatment services}

Auxiliary Devices application in swallowing disorders

UES botulinum toxin injection and myotomy
Manometry

$\begin{array}{llllll}61 & 58.1 & 37 & 35.2 & 7 & 6.7 \\ 76 & 72.4 & 24 & 22.9 & 5 & 4.8 \\ & & & & & \\ 37 & 35.2 & 57 & 54.3 & 11 & 10.5 \\ 39 & 37.1 & 52 & 49.5 & 14 & 13.3 \\ 43 & 41.0 & 50 & 47.6 & 12 & 11.4 \\ 22 & 21.0 & 39 & 37.1 & 44 & 41.9 \\ 33 & 31.4 & 45 & 42.9 & 27 & 25.7 \\ 44 & 41.9 & 47 & 44.8 & 14 & 13.3 \\ 79 & 75.2 & 23 & 21.9 & 3 & 2.9 \\ & & & & & \\ 51 & 48.6 & 42 & 40.0 & 12 & 11.4 \\ 22 & 21.0 & 50 & 47.6 & 33 & 31.4 \\ 51 & 48.6 & 44 & 41.9 & 10 & 9.5 \\ & & & & & \\ 36 & 34.3 & 56 & 53.3 & 13 & 12.4\end{array}$

$\begin{array}{llllll}33 & 31.4 & 43 & 41.0 & 29 & 27.6 \\ 25 & 23.8 & 48 & 45.7 & 32 & 30.5 \\ 19 & 18.1 & 49 & 46.7 & 37 & 35.2 \\ 57 & 54.3 & 30 & 28.6 & 18 & 17.1 \\ & & & & & \\ 14 & 13.3 & 49 & 46.7 & 42 & 40.0 \\ 14 & 13.3 & 55 & 52.4 & 36 & 34.3 \\ 64 & 61.0 & 30 & 28.6 & 11 & 10.5 \\ & & & & & \\ 15 & 14.3 & 45 & 42.9 & 45 & 42.9 \\ 20 & 19.0 & 41 & 39.0 & 44 & 41.9 \\ & & & & & \\ 44 & 41.9 & 44 & 41.9 & 17 & 16.2 \\ 41 & 39.0 & 42 & 40.0 & 22 & 21.0\end{array}$

${ }^{1}$ Low acuity service/treatment (postponing the service/treatment will not harm the patient)

${ }^{2}$ Intermediate acuity service/treatment (postponing the service/treatment will potentially harm the patient)

${ }^{3}$ High acuity service/treatment (postponing the service/treatment will harm the patient)

Table 2: Descriptive statistics; Services delivery

\begin{tabular}{lcccc}
\hline \multirow{2}{*}{ Implemented services/treatment } & \multicolumn{2}{c}{ Tele-practice } & \multicolumn{2}{c}{ Face-To-Face practice } \\
\cline { 2 - 5 } I. Voice Disorders & No. & $\%$ & No. & $\%$ \\
Non-interventional diagnostic services & & & & \\
Diagnostic interview in voice disorders & & & & \\
Perceptual evaluation of voice disorders & 84 & 80.0 & 21 & 20.0 \\
Voice-related quality of life questionnaire & 75 & 71.4 & 30 & 28.6 \\
Voice recording & 93 & 88.6 & 12 & 11.4 \\
Rehabilitative services & 80 & 76.2 & 25 & 23.8 \\
Voice therapy & & & & \\
& & & & \\
\end{tabular}




\begin{tabular}{|c|c|c|c|c|}
\hline Electrolarynx rehabilitation & 59 & 56.2 & 46 & 43.8 \\
\hline \multicolumn{5}{|l|}{ II. Speech, Language and Hearing Disorders } \\
\hline \multicolumn{5}{|l|}{ Non-interventional diagnostic services } \\
\hline Diagnostic interview $^{1}$ & 88 & 83.8 & 17 & 16.2 \\
\hline Developmental questionnaires & 95 & 90.5 & 10 & 9.5 \\
\hline Evaluation of verbal and non-verbal communication & 64 & 61.0 & 41 & 39.0 \\
\hline Evaluation of speech perception and production & 67 & 63.8 & 38 & 36.2 \\
\hline Estimation of cognitive and developmental age & 62 & 59.0 & 43 & 41.0 \\
\hline Evaluation of reading and writing & 64 & 61.0 & 41 & 39.0 \\
\hline \multicolumn{5}{|l|}{ Rehabilitative services } \\
\hline Language and speech therapy & 67 & 63.8 & 38 & 36.2 \\
\hline Rehabilitation of hearing disabled & 45 & 42.9 & 60 & 57.1 \\
\hline Augmentative and alternative communication & 61 & 58.1 & 44 & 41.9 \\
\hline \multicolumn{5}{|l|}{ III. Swallowing disorders } \\
\hline \multicolumn{5}{|l|}{ Non-interventional diagnostic services } \\
\hline Diagnostic interview in swallowing disorders & 68 & 64.8 & 37 & 35.2 \\
\hline Bedside evaluation of swallowing & 26 & 24.8 & 79 & 75.2 \\
\hline Nutritional evaluation & 54 & 51.4 & 51 & 48.6 \\
\hline Swallowing-related quality of life questionnaires & 84 & 80.0 & 21 & 20.0 \\
\hline \multicolumn{5}{|l|}{ Rehabilitative services } \\
\hline Swallowing therapy & 54 & 51.4 & 51 & 48.6 \\
\hline Dietetic and nutritional therapy & 76 & 72.4 & 29 & 27.6 \\
\hline
\end{tabular}

${ }^{1}$ Including family tree tracing in familial hearing disorders

\section{Classification of patients according to morbidity:}

Most respondents classified dysphonic patients with airway compromise as urgent patients (56, 53.3\%), whereas patients without airway compromise and phonasthenic patients were considered elective (43, 41\%) and $(85,81 \%)$ respectively (Table 3$)$. Patients receiving regular botulinum toxin treatment and laryngectomees were mostly classified as semi-elective $(56,53.3 \%)$, and $(51,48 \%)$, respectively. Similarly, patients with dysarthria, aphasia or dysphasia, and childhood hearing disorders
$(60,57 \%),(59,56.2 \%)$, and $(55,52.4 \%)$ respectively. However, patients with developmental language delay, resonance disorders, reading and writing disorders, and lastly, childhood and adulthood fluency disorders were mostly considered elective.

The majority classified patients with swallowing/ feeding disorders whether childhood or adulthood post-traumatic and stroke patients as urgent patients $(60,57 \%), \quad(57,54.3 \%), \quad$ and $(66$ $62.9 \%$ ) respectively.

Table 3: Classification of patients according to morbidity

\begin{tabular}{|c|c|c|c|c|c|c|c|c|}
\hline \multirow[t]{2}{*}{ Patients/complaints } & \multicolumn{2}{|c|}{$\begin{array}{l}\text { Elective } \\
\text { patient }^{1}\end{array}$} & \multicolumn{2}{|c|}{$\begin{array}{c}\text { Semi-elective } \\
\text { patient }^{2}\end{array}$} & \multicolumn{2}{|c|}{$\begin{array}{c}\text { Semi-urgent } \\
\text { patient }^{3}\end{array}$} & \multicolumn{2}{|c|}{ Urgent patient ${ }^{4}$} \\
\hline & No. & $\%$ & No. & $\%$ & No. & $\%$ & No. & $\%$ \\
\hline \multicolumn{9}{|l|}{ I. Voice Disorders } \\
\hline Dysphonia with airway compromise & 19 & 18.1 & 13 & 12.4 & 17 & 16.2 & 56 & 53.3 \\
\hline Dysphonia without airway compromise & 43 & 41.0 & 43 & 41.0 & 15 & 14.3 & 4 & 3.8 \\
\hline Phonasthenia & 85 & 81.0 & 18 & 17.1 & 2 & 1.9 & 0 & 0.0 \\
\hline $\begin{array}{l}\text { Patients receiving regular botulinum toxin injection for voice } \\
\text { spasm, tremors or VCD }\end{array}$ & 23 & 21.9 & 56 & 53.3 & 18 & 17.1 & 8 & 7.6 \\
\hline $\begin{array}{l}\text { Laryngectomees receiving Provox electrolarynx, oesophageal } \\
\text { speech or voice prosthesis }\end{array}$ & 34 & 32.4 & 51 & 48.6 & 16 & 15.2 & 4 & 3.8 \\
\hline
\end{tabular}


II. Speech, language, and hearing disorders

Developmental language delay

Acquired language disorders (aphasia or dysphasia)

Dysarthria

Resonance disorders

Reading and writing abnormalities

Childhood fluency disorders

Childhood hearing disorders

Adulthood fluency disorders

$\begin{array}{lccccccc}56 & 53.3 & 45 & 42.9 & 2 & 1.9 & 2 & 1.9 \\ 31 & 29.5 & 59 & 56.2 & 13 & 12.4 & 2 & 1.9 \\ 33 & 31.4 & 60 & 57.1 & 12 & 11.4 & 0 & 0.0 \\ 49 & 46.7 & 48 & 45.7 & 8 & 7.6 & 0 & 0.0 \\ 69 & 65.7 & 34 & 32.4 & 2 & 1.9 & 0 & 0.0 \\ 58 & 55.2 & 43 & 41.0 & 4 & 3.8 & 0 & 0.0 \\ 19 & 18.1 & 55 & 52.4 & 27 & 25.7 & 4 & 3.8 \\ 52 & 49.5 & 47 & 44.8 & 6 & 5.7 & 0 & 0.0 \\ & & & & & & & \\ 4 & 3.8 & 10 & 9.5 & 31 & 29.5 & 60 & 57.1 \\ 4 & 3.8 & 8 & 7.6 & 36 & 34.3 & 57 & 54.3 \\ 5 & 4.8 & 10 & 9.5 & 24 & 22.9 & 66 & 62.9\end{array}$

III. Swallowing disorders

Early childhood feeding or swallowing difficulties or failure to thrive

Post-traumatic or post-operative feeding or swallowing difficulties

Acute cases of dysphagia including ICU and stroke patients

${ }^{1}$ Patients without significant morbidity

${ }^{2}$ Patients with significant morbidity if not corrected within 3-6 months

${ }^{3}$ Patients with significant morbidity if not corrected within 48-72 hours

${ }^{4}$ Patients with significant morbidity if not corrected immediately

\subsection{Association between demographic characteristics and the overall score of implemented services, the score of patients' morbidity:}

The differences in implemented services grading were significantly related to the gender, type of working institution, and inclusion of working institutions to treat COVID-19 cases. Whilethe differences in gender and age significantly influenced respondents' classification of patients/complaints (Table 4).

\subsection{Association between service delivery and the} overall score of implemented services grading, the

\section{score of patients' morbidity:}

The face-to-face practice was a significantly more likely approach to swallowing, dietetic and nutritional therapies than tele-practice. Likewise, it was a significant approach to handle critical patients requiring swallowing therapy (Table 5).

\subsection{Correlation between the overall score of implemented services grading and score of patients' morbidity:}

Respondents' grading of implemented services was positively correlated tothe degree of patient morbidity (Figure1).

Table 4: Association between demographic characteristics and the overall score of implemented services grading, score of patients' morbidity classification

\begin{tabular}{|c|c|c|c|c|}
\hline & $\begin{array}{l}\text { Implemented } \\
\text { services score }\end{array}$ & \multirow[t]{2}{*}{$P$ value } & $\begin{array}{c}\text { Score of } \\
\text { patients'morbidity }\end{array}$ & \multirow[t]{2}{*}{$P$ value } \\
\hline & Mean \pm SD & & Mean \pm SD & \\
\hline $\begin{array}{l}\text { Gender } \\
\text { Male } \\
\text { Female }\end{array}$ & $\begin{array}{l}70.07 \pm 12.49 \\
78.62 \pm 14.84\end{array}$ & $0.037^{*}$ & $\begin{array}{l}29.93 \pm 4.33 \\
34.29 \pm 7.09\end{array}$ & $0.023 *$ \\
\hline $\begin{array}{l}\text { Age: (years) } \\
\quad<40 \\
\quad \geq 40\end{array}$ & $\begin{array}{l}76.01 \pm 14.73 \\
79.84 \pm 14.74\end{array}$ & 0.204 & $\begin{array}{l}32.54 \pm 6.89 \\
35.66 \pm 6.59\end{array}$ & $0.026^{*}$ \\
\hline $\begin{array}{l}\text { Current social sta } \\
\text { Married } \\
\text { Not-married }\end{array}$ & $\begin{array}{l}77.06 \pm 14.22 \\
80.27 \pm 19.52\end{array}$ & 0.498 & $\begin{array}{l}33.46 \pm 6.83 \\
35.45 \pm 7.72\end{array}$ & 0.367 \\
\hline $\begin{array}{l}\text { Number of child } \\
\text { No } \\
\text { Yes }\end{array}$ & $\begin{array}{l}77.07 \pm 15.08 \\
77.46 \pm 14.81\end{array}$ & 0.925 & $\begin{array}{l}35.73 \pm 6.10 \\
33.32 \pm 7.01\end{array}$ & 0.213 \\
\hline
\end{tabular}




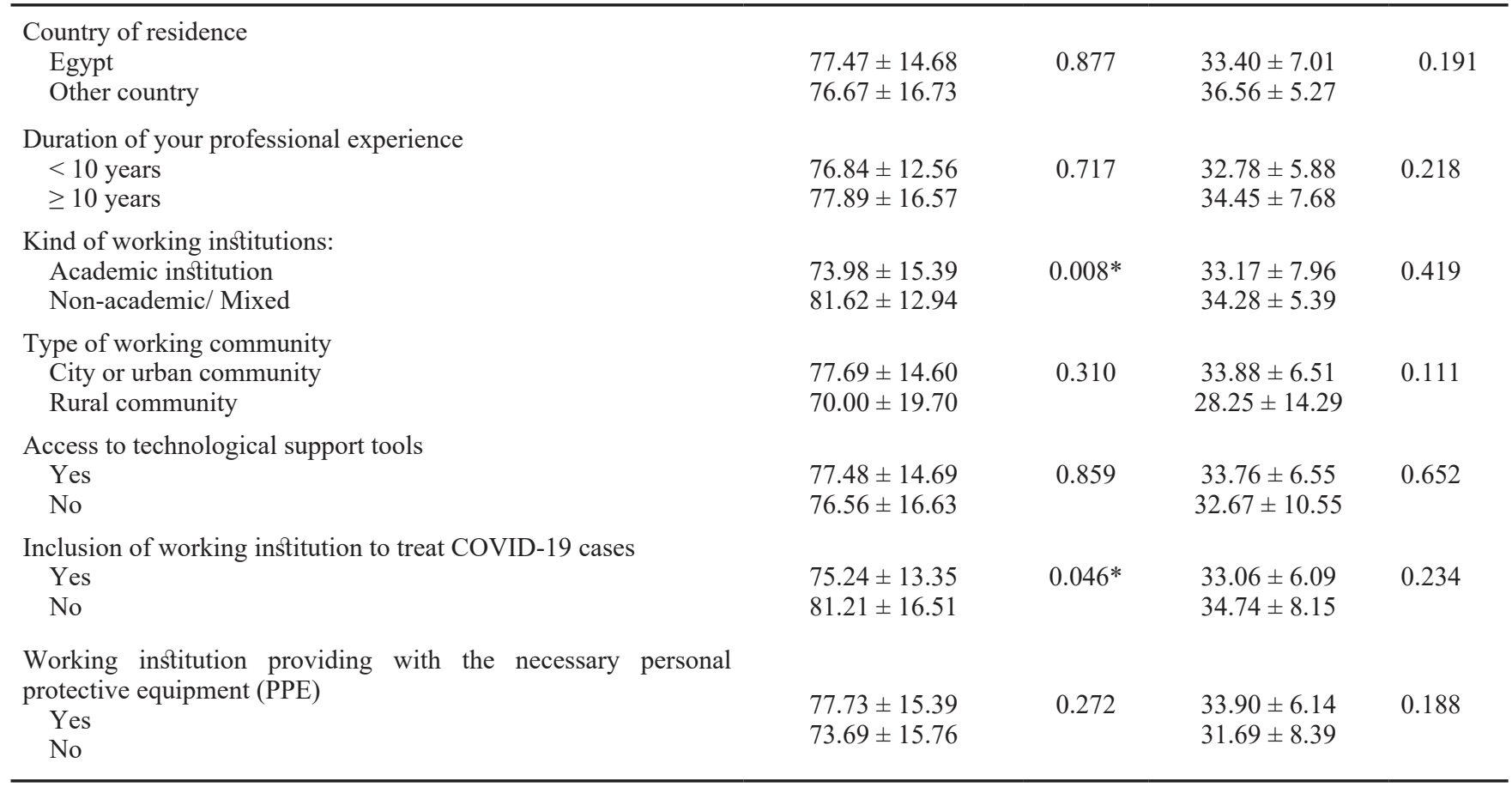

Test used: Independent sample T-test

* Significant $P$-value $<0.05$

Table 5: Association between service delivery and the overall score of implemented services grading, score of patients' morbidity classification

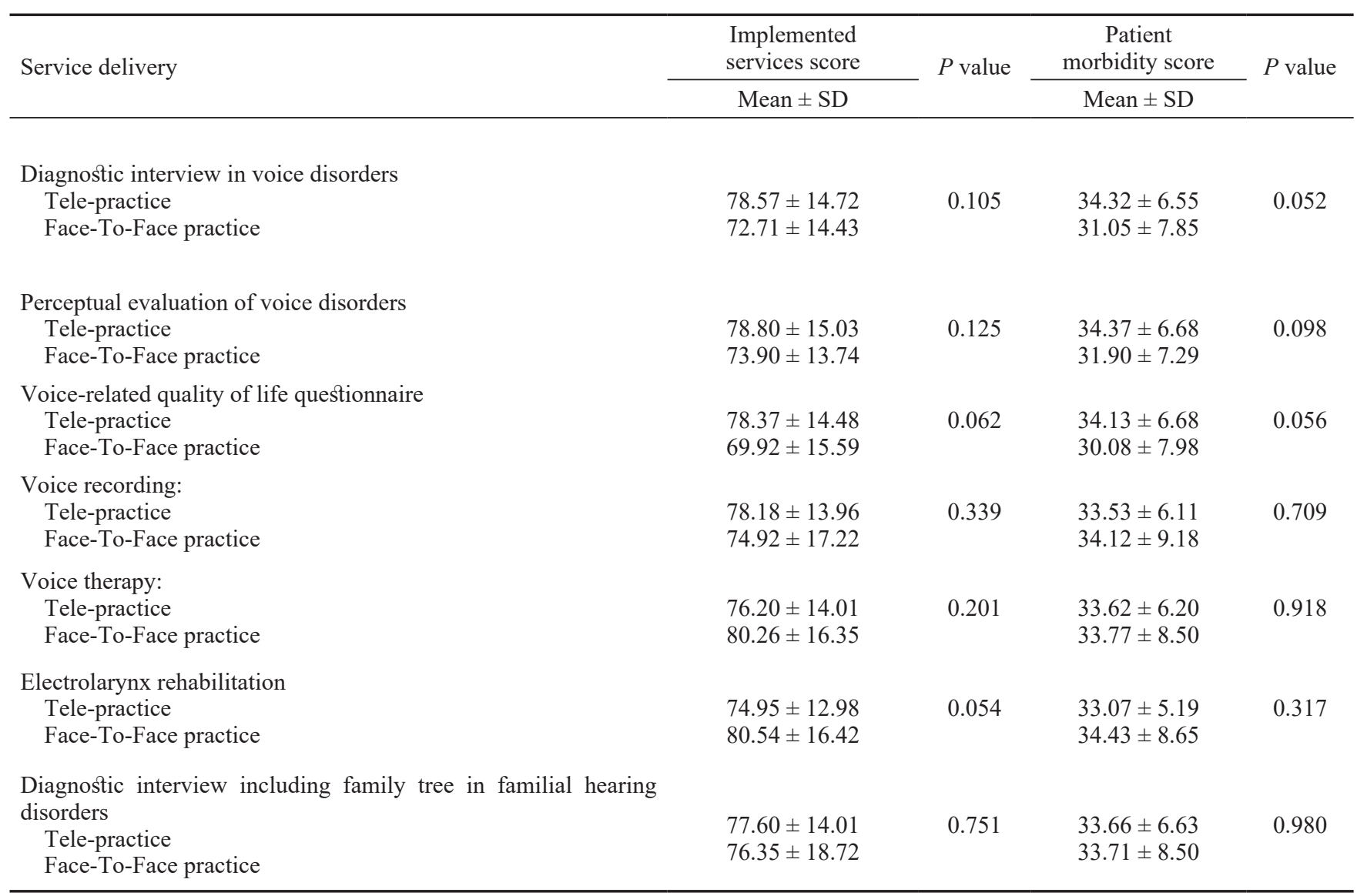




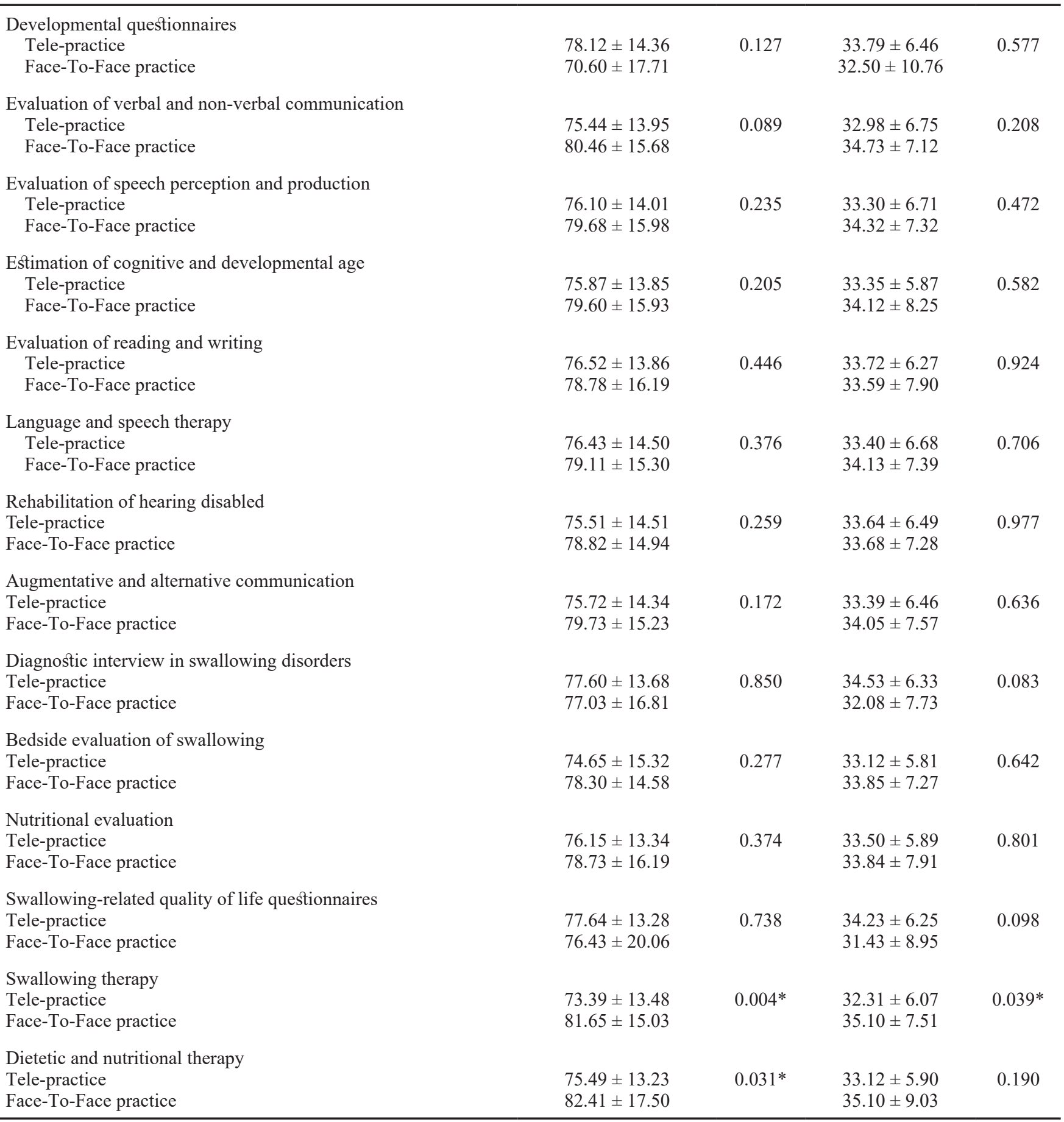

Test used: Independent sample T-test

* Significant $P$-value $<0.05$ 


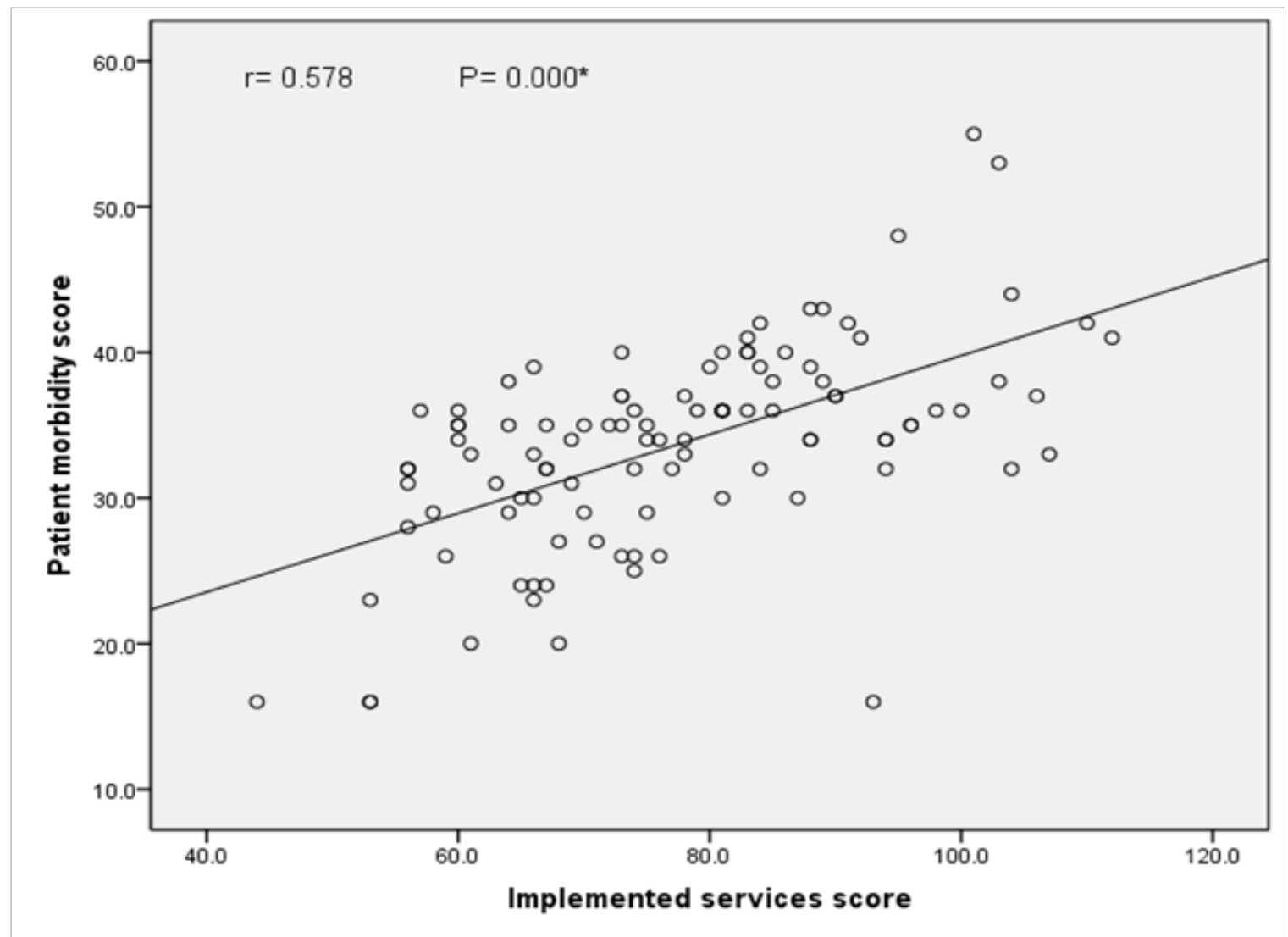

Fig. 1: Correlation between the overall score of implemented services grading and the score of patients' morbidity

\section{DISCUSSION}

Phoniatricians are not among the frontline health care professionals in caring for patients during infectious outbreaks. Nevertheless, this practice entails providing clinical services to patients with upper airway disorders, swallowing, and feeding disorders who might be at a considerable risk if the clinical services' suspended or postponed. Guidelines for Phoniatric practice during the COVID-19 pandemic are still lacking. In this study, we used a survey as a measure of phoniatricians' practices during the pandemic.

Our survey shows that most participants agree that the non-interventional diagnostic measures of voice disorders, speech, and language and hearing disorders can be safely postponed. The results were incongruent with the UEP statement expressing that these measures can be done through tele-consultation ${ }^{[3]}$. Still, our data show that similar measures of swallowing disorders can be undertaken with precautions agreeing with the recommendations of the UEP statement ${ }^{[3]}$ and in fact anticipated given the potential complications of oropharyngeal dysphagia as aspiration pneumonia and malnutrition.

Interventional diagnostic procedures of voice and swallowing disorders, including trans-oral rigid or transnasal flexible laryngoscopies, are among the AGPs that carry the risk of infection transmission. Thus concerns have to be taken to balance the risk of infection transmission and patients' complications, which is illustrated in our survey. Most respondents consider them intermediate acuity services, which supports the available corresponding guidelines and recommendations ${ }^{[3-7]}$. The same applies to the survey results concerning the interventional diagnostics of speech, language, and hearing disorders however, contrasting with the UEP statement that declared that hearing examinations and assessment should be executed promptly ${ }^{[3]}$. A possible explanation is that most of our responses are received from phoniatricians residing in Egypt where Phoniatrics and Pediatric audiology are separate specialties. Nevertheless, our survey confirms that newborn hearing screening is a high acuity service agreeing with the UEP statement that emphasized the same point ${ }^{[3]}$.

In line with the international recommendations ${ }^{[3]}$, we found that the instrumental voice, speech, language disorders diagnostics, especially for patients with elective scenarios, can be postponed since they are also considered potential AGPs.

While, the UEP recommendations highlighted that laryngeal procedures/surgeries, including office-based vocal fold injection, can be performed after prior SARSCov-2 testing ${ }^{[3]}$, our data describe that these procedures can 
be delayed. This is Likely due to the restriction of testing in Egypt to the suspected cases during the current survey ${ }^{[8]}$. However, our results agree with the guidelines introduced by the American Academy of head and neck surgery which reported the consideration of postponing these procedures for more than 30 days $^{[6]}$.

Contrary to the UEP recommendations that advocated the delivery of language, and speech therapy in the presence of special protective precautions [3], our survey recommends postponing them. This may be due to the anticipated less adherence of Egyptians to the various protective precautions ${ }^{[9]}$. Nevertheless, our findings support the UEP recommendations that stipulated that rehabilitation of the hearing disabled should be prioritized. Our results advocate postponing voice therapy while the UEP recommendations embraced the use of remote voice therapy ${ }^{[3]}$. This variation is possibly due to the lack of patients' awareness of tele-medicine in Egypt ${ }^{[10]}$.

ENT and Phoniatric societies' recommendations ${ }^{[2-7,12]}$, implied that clinical service delivery should be transferred more to the tele-practice patterns. This was further supported by our data showing that tele-practice is a suitable approach for service delivery during pandemics.

In the context of the COVID-19 pandemic, it was crucial to triage patients/complaints according to potential morbidity. This is reflected by our survey, which reveals that most Phoniatric patients are regarded as elective except for patients with airway compromise and those with swallowing disorders are considered urgent patients. These findings are concordant with the corresponding recommendations ${ }^{[4-7,12]}$. Whereas, patients receiving regular botulinum toxin treatment, laryngectomees, and patients with dysarthria and aphasia are considered semielective. The latter responses are owed to the concerns for the increased risk of transmission of the virus with close examinations/procedures of the head and neck ${ }^{[4-7]}$.

Interestingly, our data show that females are significantly inclined to advance services. In contrast, males tend to postpone them, supporting the opinion stating that physicians' dedication toward their patients during pandemics is not restricted to the male gender ${ }^{[11]}$. While respondents working in academic institutions consider most Phoniatric services to be intermediate acuity, those working in non-academic institutions tend to consider them higher acuity. This is true as most academic institutions are currently directed toward managing patients with COVID-19, which consumes most of their resources; thus, managing patients with elective or semielective scenarios has been deferred ${ }^{[4,12,13]}$. Furthermore, respondents working in institutions treated COVID-19 cases were significantly more likely to postpone most of the services than those whose institutions did not treat
COVID-19 cases. Institutional priority to treat COVID-19 cases probably contributed to the later findings ${ }^{[12]}$.

Additionally, phoniatricians more than 40 years old are significantly more likely to triage patients to be critical than phoniatricians less than 40 years (35.66 vs. $32.54, P<0.026)$. The duration of professional experience maybe a plausible cause of this finding. Female phoniatricians are significantly more likely to triage patients to be more morbid than male phoniatricians. This may be because most of our survey's respondents were female. It is noteworthy that this is the first formal Phoniatric guideline to associate between demographic characteristics and patient triaging during the COVID-19 pandemic.

Despite the current pandemic situation, face-toface practice is a significantly more likely approach for swallowing, dietetic, and nutritional therapy than telepractice. Similarly, it is a significant approach to handle urgent patients requiring swallowing therapy. These findings are consistent with the available guidelines and recommendations ${ }^{[3-7]}$ and maybe attributed to the urgency of swallowing rehabilitation that necessitates patient confrontation.

The positive relationship between the implemented services and patients' morbidity grading is an expected outcome that reinforces the recommendations advocated that Phoniatric and/or ENT services should be prioritized to urgent patients ${ }^{[4-7]}$.

This survey was conducted during the first wave of the COVID-19 pandemic. At that time, it was a critical situation that confronted all health care providers, including phoniatricians who generally deal with patients with less acute pathology; nonetheless, examinations/treatments are challenging to execute remotely. We provided a riskassessment framework that guides Phoniatricians to a safer practice without affecting patients' survival or recovery.

The only limitation is that most of the responses were from Egypt, with a few responses from other areas of the world. However, it is essential to note that Egypt's Phoniatric specialty is well established and assembles many well-experienced phoniatricians. Nevertheless, we intend to repeat the questionnaire and include a larger group of phoniatricians to investigate service implementation adjustments in the post-vaccination period.

\section{CONCLUSION}

We provided evidence-based guidelines of Phoniatric service implementation during the current or future infective outbreak. Most Phoniatric services can be safely postponed except for voice and swallowing disorders diagnostics and treatments. Dysphonic patients with airway compromise and patients with swallowing or feeding 
difficulties are considered urgent patients. A paradigm shift to tele-practice is recommended.

\section{ACKNOWLEDGMENT}

We gratefully acknowledge the support of the Egyptian Society of Phoniatrics and Logopedics (ESPL) for assistance in obtaining the data of the questionnaire. We also acknowledge Prof. Nirvana Gamal-Eldin (Ain Shams University), Prof. Sahar Shohdy (Cairo University), and Prof. Amal Elsaeed (Zagazig University) for their valuable comments in reviewing the questionnaire-based survey of the current study.

\section{CONFLICT OF INTEREST}

There are no conflicts of interest

\section{REFERENCES}

1. Saadi RA, Bann DV, Patel VA, et al. A Commentary on Safety Precautions for Otologic Surgery during the COVID-19 Pandemic.Otolaryngol-Head and Neck Surg2020;162(6): 797-99.

2. Patel ZM, Fernandez-Miranda J, Hwang PH, Nayak JV, Dodd R, Sajjadi H, et al. Precautions for endoscopic transnasal skull base surgery during the COVID-19 pandemic.Neurosurgery 2020.

3. Geneid A, Nawka T, Schindler A, et al. Union of the European Phoniatricians' position statement on the exit strategy of phoniatric and laryngological services: staying safe and getting back to normal after the peak of coronavirus disease 2019. J LaryngolOtol 2020; 1-10.

4. Lescanne E, Van der Mee-Marquet N, Juvanon $\mathrm{J}-\mathrm{M}$, et al. Best practice recommendations: ENT consultations during the COVID-19 Pandemic. Eur Ann of OtolRhinollaryngol, Head and Neck diseases 2020; 1064; 1-6.

5. Setzen G, Anne S, Brown III EG, et al. Guidance for Return to Practice for Otolaryngology Head and Neck Surgery: Part One: American Academy of
Otolaryngology-Head and Neck Surgery (AAO-HNS); May 5, 2020 Retrieved fromhttps://www.entnet. $\mathrm{org} / \mathrm{sites} /$ default/files/uploads/guidance for_return to_practice_part_one final 050520.pdf accessed $12 \mathrm{Feb} 2021$.

6. Setzen G, Anne S, Brown III EG, et al. Guidance for Return to Practice for Otolaryngology- Head and Neck Surgery: Part two: American Academy of Otolaryngology Head and Neck Surgery (AAOHNS); May 12, 2020 updated July 1, 2020. Retrieved from https://www.entnet.org/sites/default/files/ uploads/guidance_for_return_to_practice part one final 050520.pdf accessed 12 Feb 2021.

7. Vukkadala N, Qian Z J, Holsinger F C, Patel Z M, Rosenthal E. COVID-19 and the otolaryngologist preliminaryevidence-basedreview.Laryngoscope2020 Nov;130(11): 2537-2543. Doi: 10.1002/lary.28672.

8. Masoud HH, Elassal G, Zaky S, et al. Management Protocol for COVID-19 Patients Version 1.4/30 May 2020 Ministry of health and population (MOHP), Egypt.

9. Hakim SA, Abouelezz NF, El Okda EM. Use OF Personal Protective Devices Among health care workers in a teaching hospital in Cairo, Egypt.Egypt $\mathrm{J}$ of Occup Med, 2016; 40 (2):287-300.

10. El Tokali S, Fishta A, Shams K. Saving children through tele-consultation in remote Egypt. eHealth Magazine. 2009 Dec [cited 2011 June 16]. Available from: http://ehealth.elestonline.com/2009/12/11353/

11. Brubaker L, Women Physicians and the COVID-19 Pandemic. Opinion.JAMA September 1, 2020; 324(9): 835-836.

12. Tysome J R, Bhutta M F. COVID-19: Protecting our ENT Workforce. Clinical Otolaryngology.2020; 45: 311-312. DOI: 10.1111/coa.13542.

13. Christopher C, William B A, Burton V R, Dan G B. COVID-19: Financial Stress Test for Academic Medical Centers. Academic Medicine: August 2020. 95(8); 1143-1145. Doi: 10.1097/ACM.0000000000003418. 